\title{
Geomagnetic reversal frequency since the Late Cretaceous
}

\author{
William Lowrie ${ }^{1}$ and Dennis V. Kent ${ }^{2}$ \\ ${ }^{I}$ Institut für Geophysik, E.T.H. - Hönggerberg, CH-8093 Zürich (Switzerland) \\ ${ }^{2}$ Lamont -Doherty Geological Observatory, Palisades, NY 10964 (U.S.A.)
}

Received September 21, 1982

Revised version accepted December 12, 1982

\begin{abstract}
Models of geomagnetic reversals as a stochastic or gamma renewal process have generally been tested for the Heirtzler et al. [1] magnetic polarity time scale which has subsequently been superseded. Examination of newer time scales shows that the mean reversal frequency is dominated in the Cenozoic and Late Cretaceous by a linearly increasing trend on which a rhythmic fluctuation is superposed. Subdivision into two periods of stationary behavior is no longer warranted. The distribution of polarity intervals is visibly not Poissonian but lacks short intervals. The LaBrecque et al. [2] polarity time scale shows the positions of 57 small-wavelength marine magnetic anomalies which may represent short polarity chrons. After adding these short events the distribution of all polarity intervals in the age range $0-40 \mathrm{Myr}$ is stationary and does not differ significantly from a Poisson distribution. A strong asymmetry develops in which normal polarity chrons are Poisson distributed but reversed polarity chrons are gamma distributed with index $k=2$. This asymmetry is of opposite sense to previous suggestions and results from the unequal distribution of the short polarity chrons which are predominantly of positive polarity and concentrated in the Late Cenozoic. If short-wavelength anomalies arise from polarity chrons, the geomagnetic field may be more stable in one polarity than the other. Alternative explanations of the origin of short-wavelength marine magnetic anomalies cast doubt on the inclusion of them as polarity chrons, however. The observed behavior of reversal frequency suggests that core processes governing geomagnetic reversals possess a long-term memory.
\end{abstract}

\section{Introduction}

The stability of physical processes in the earth's fluid outer core is expressed in the spectrum of geomagnetic field behavior. The long-term stability, in particular, can be inferred from the past history of magnetic polarity reversals. The longest, continuous record of polarity history comes from the interpretation of marine magnetic anomalies. Of particular interest is the episode of reversal behaviour which began at the end of the Cretaceous quiet interval in the earliest Campanian and which persists until the present. Analysis of this reversal sequence has led to several suggested mag-

Institut für Geophysik, E.T.H. Zürich, Contribution No. 398. Lamont-Doherty Geological Observatory, Contribution No. 3411 . netic polarity time scales for the Late Cretaceous and Cenozoic.

The first polarity time scale for this entire interval was deduced by Heirtzler et al. [1]. After comparing marine magnetic profiles in different ocean basins they concluded that sea-floor spreading was most likely to have remained constant in the South Atlantic, and selected profile Vema-20 as the basis of their magnetic polarity time scale. This time scale has undergone several modifications as more restrictive data became available. LaBrecque et al. [2] made various adjustments to the reversal sequence to bring their occurrence and the lengths of polarity zones in line with current knowledge. Ness et al. [3] summarized various other evolutionary modifications to the polarity time scale and recomputed anomaly ages. Recently, the results of magnetostratigraphic investi- 
gations in Italian limestone sections have been incorporated in a further revision of the magnetic polarity time scale for the Late Cretaceous and Cenozoic [4].

As a result of the discovery of additional wellcorrelated anomalies, the deletion of suspect ones such as anomaly 14, and improved dating of the Late Cretaceous and Cenozoic anomaly sequence the Heirtzler et al. [1] time scale has been superseded by newer versions which are truer representations of geomagnetic polarity history. These successive revisions have involved substantial changes. It is, therefore, opportune to re-examine some of the statistical properties of geomagnetic reversal behavior that were deduced from the Heirtzler et al. [1] time scale.

\section{Statistical analysis of the magnetic polarity time scale}

A major feature of the Heirtzler et al. [1] reversal time scale which has been recognized in all previous analyses [5-7] is an abrupt, threefold increase in the average frequency of reversals at about 45 Myr B.P. (Fig. 1a); the mean length of polarity intervals decreases from 0.90 to $0.33 \mathrm{~m}$.y. [7]. Cox [8] suggested that this discontinuity divides the time scale into two intervals of apparently stationary behavior of the geomagnetic dynamo, each characterized by different statistical properties. Analyses of reversal frequency and of the lengths of polarity intervals usually have treated the intervals before and after this discontinuity separately $[5,6]$.

For statistical analysis of a time series to be valid the series must be stationary. A necessary condition for this is that there must be no significant time-dependent trend in the data. Naidu [6] found that the polarity interval distribution was stationary in the 0-48 Myr and 56-72 Myr parts of the Heirtzler et al. [1] time scale. The stationary character of the reversal frequency in the age range $0-48 \mathrm{Myr}$ is also illustrated in Fig. la. The points in this figure represent moving averages over an $8-\mathrm{Myr}$ window. Every eighth point is independent and can be used in linear regression analysis. The slope of the regression line can be

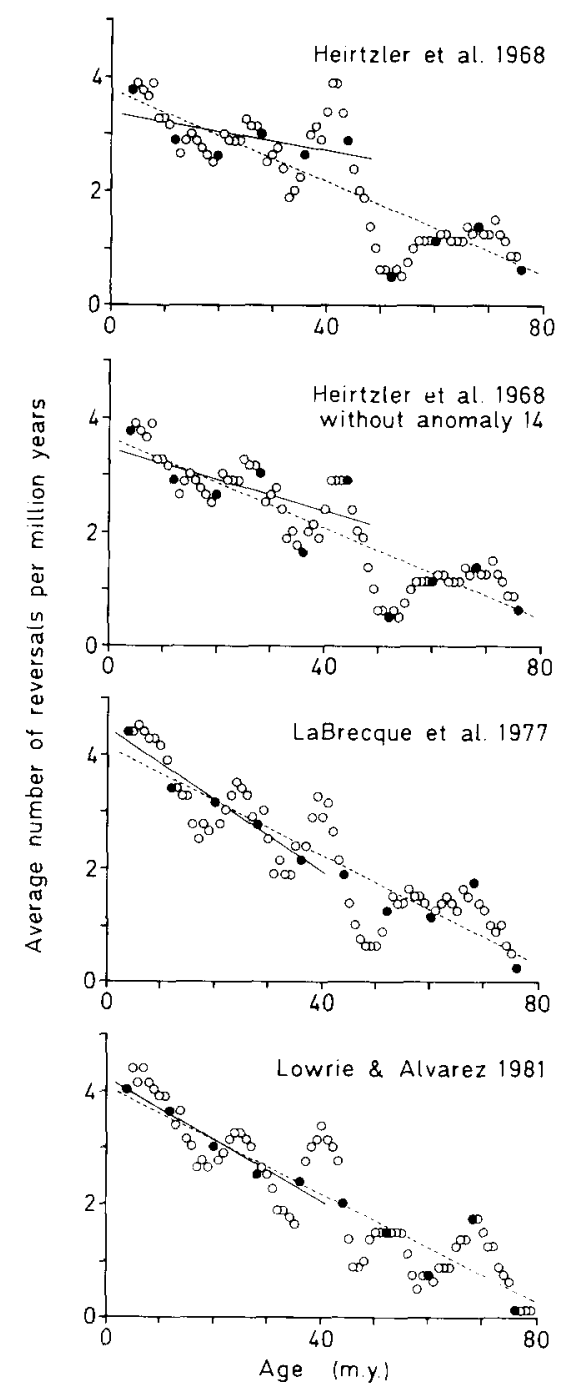

Fig. 1. Variation with age of the average reversal frequency, computed in an 8-Myr moving window, for several magnetic polarity time scales. In each case the dotted line is the linear regression of the independent eighth (solid) points; the solid line is a linear regression for $0-48 \mathrm{Myr}$ in (a) and (b) and for $0-40 \mathrm{Myr}$ in (c) and (d).

tested with a $t$-statistic under the assumption that the deviations from linearity approximate to a Gaussian distribution, and is found to be not significantly different from zero at the $95 \%$ confidence level.

Since 1968 the appearance of the magnetic time scale has been altered in a number of significant 
ways. A major difference results when anomaly 14 is omitted. This anomaly in the Heirtzler et al. [1] sequence is not found on most marine magnetic profiles and was dropped from later time scales. Anomaly 14 represents four very short normal polarity zones and introduces eight additional reversal boundaries, spuriously increasing average reversal frequency in the 35-45 Myr age interval. Without this anomaly the 45-Myr "discontinuity" is partially reduced and the linear regression for 0-48 Myr is more poorly correlated, although the slope of the linear trend for this part of the time scale is still not significantly different from zero at the 95\% confidence level (Fig. lb).

The 65-Myr age of the Cretaceous/Tertiary boundary places it just older than anomaly 26 in the Heirtzler et al. [1] time scale. In Italian magnetostratigraphic studies this boundary was located between anomalies 29 and $30[9,10]$. Polarity interval lengths in the Late Cretaceous were revised using North Pacific marine magnetic profiles [11] and the definition of the reversal sequence between anomalies 5 and 6 was improved as a result of the acquisition of more and better marine magnetic data. LaBrecque et al. [2] incorporated these improvements in a revised polarity time scale. Subsequently, magnetostratigraphic research has located most of the Paleogene stage boundaries in the reversal sequence [12]. These correlations have been used in a recent revision of the polarity time scale [4], which gives improved agreement between magnetic anomaly ages and DSDP basal sediment ages. However, the major differences from the Heirtzler et al. [1] time scale were to the largest part achieved in the LaBrecque et al. [2] revision and the later improvement in calibration does not greatly alter statistical properties of the time scales.

When these time scales are re-examined after making the mentioned changes, two principal features can be distinguished. The average reversal frequency appears to be characterized by a fluctuation about an almost linearly increasing trend for the past $80 \mathrm{Myr}$. The slope of this trend for the Cenozoic and Late Cretaceous anomaly sequence is significantly different from zero for all the magnetic polarity time scales studied in their entirety (Fig. 1). In the revised time scales of LaBrecque et al. [1] and Lowrie and Alvarez [4]

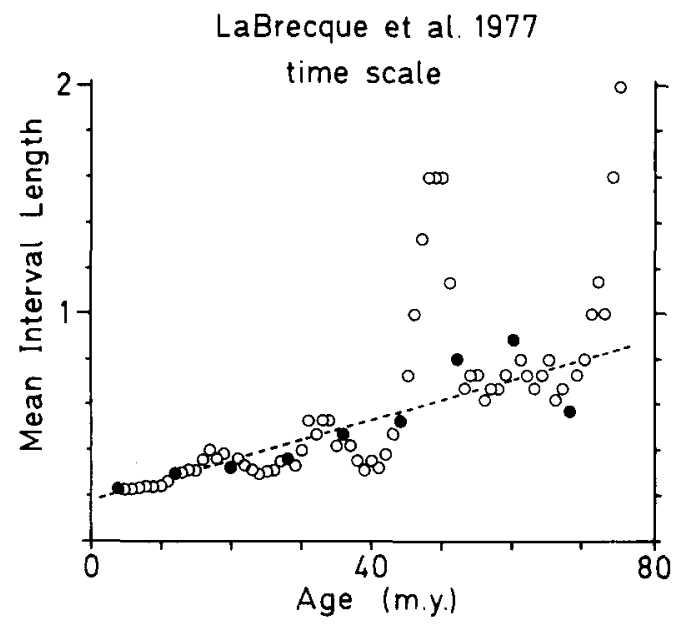

Fig. 2. Variation with age of the average polarity interval length in the LaBrecque et al. [2] magnetic polarity time scale, computed in an 8-Myr moving window.

the trend is also significant in the range $0-40 \mathrm{Myr}$.

Statistical analyses of reversal behaviour have been usually based upon the distribution of lengths of polarity intervals. The average number of reversals per million years, calculated for a moving window of width $8 \mathrm{Myr}$ for the polarity time scale of LaBrecque et al. [2], is shown in Fig. 2. Analysis of the independent eighth values again gives a trend that is significant at the $95 \%$ level of confidence. Corresponding statistical examination of the Lowrie and Alvarez [4] time scale shows that it also is non-stationary.

\section{Statistical models of geomagnetic reversals}

The occurrence of polarity reversals has been described by different statistical models. Cox [13] assumed a stochastic model in which the probability of a reversal per unit time is constant, implying that the core has no long-term memory for earlier reversals. He found that the distribution of polarity interval lengths could be represented by a binomial distribution, which, for the case that the probability of an individual reversal is low, becomes a Poissonian distribution. Cox [5] compared the observed distributions of polarity interval lengths against a Poissonian distribution. They 
agreed well for the past 10.6 Myr but for 10.6-45 Myr B.P. the observed number of short polarity intervals was fewer than predicted by a Poissonian distribution and this discrepancy was very large for times older than $45 \mathrm{Myr}$.

Naidu [6] showed that the lengths of polarity intervals are better described by a gamma distribution, in which the probability $p(\mathrm{~d} T)$ of an interval of duration $\mathrm{d} T$ is given by:

$p(\mathrm{~d} T)=\frac{(k / m)^{k}}{\Gamma(k)} \mathrm{d} T^{k-1} \mathrm{e}^{-(k / m) \mathrm{d} T}$

Here, $m$ is the mean polarity interval length and $k$ is the gamma index.

Naidu [6] gives a simple method for determining the value of $k$ for a stationary gamma distribution. He showed that:

$\ln k-\Psi(k)=\ln m-(1 / N) \sum_{i}^{N} \ln \mathrm{d} T_{i}$

where $\Psi(k)$ is the digamma function. The righthand side of the equation is determined from the observations; the left-hand side can be graphed, and in this way the value of $k$ can be determined.

A Poissonian distribution is described by $k=1$; larger $k$-values refer to distributions in which the number of short intervals is fewer than in the Poissonian distribution. A gamma renewal process implies that the probability per unit time of a reversal occurring is not constant, and that the occurrence of each reversal is not independent of earlier ones.

Naidu [6] showed that polarity chrons in the 0-48 Myr B.P. part of the Heirtzler et al. [1] time scale are fitted best by a gamma distribution with $k=2$; polarity chrons older than $56 \mathrm{Myr}$ were fitted best by a gamma distribution having $k=3.6$. His analysis showed a singularity at $48-56 \mathrm{Myr}$, which is also evident in Fig. 2. Phillips [7] devised a new technique for statistical analysis of the reversal sequence; this used a moving window of variable time-width in which polarity intervals were weighted with coefficients derived from a Gaussian "bell-type" distribution. He found no major difference in $k$ at the 45-Myr discontinuity but the average value of $k$ was 1.72 for the entire Heirtzler et al. [1] time scale. Phillips [7] also found that the statistics of normal polarity intervals differed significantly from those of reversed polarity intervals; the gamma index for the normal distribution was $k=2.28$ and that for the reversed distribution was $k=1.19$.

The LaBrecque et al. [2] and Lowrie and Alvarez [4] polarity time scales contain no events shorter than $40 \mathrm{kyr}$. The distributions of polarity interval lengths for both normal and reversed states in these time scales are visibly deficient in short intervals (Fig. 3). The mean length of a polarity chron was found to be non-stationary over the time interval 0-40 Myr in both time scales. Statistical properties have only limited meaning, but for qualitative comparison the mean durations and the corresponding gamma indices are listed in Table 1. In both time scales the mean length of normal chrons is less than that of reversed chrons, and the gamma index for normal chrons is larger than that for reversed chrons. For each distribution the overall gamma index is closer to 2 than to 1 .

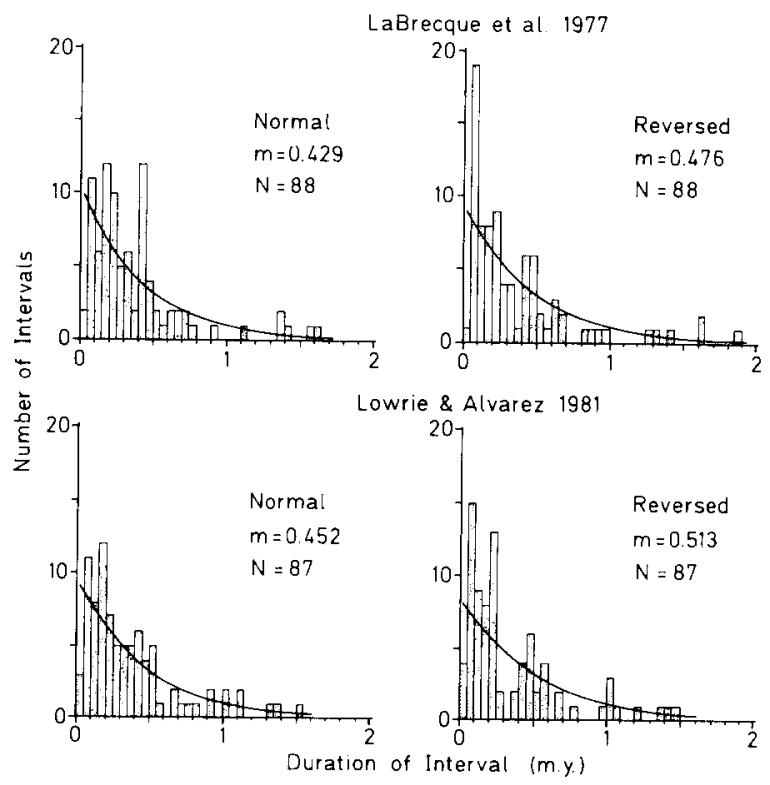

Fig. 3. The distributions of normal and reversed polarity interval lengths in the magnetic polarity time scales of (a) LaBrecque et al. [2] and (b) Lowrie and Alvarez [4]. Each distribution is lacking in short polarity intervals compared to a Poisson distribution (solid curve). Age interval: 0-80 Myr B.P. 
TABLE 1

Comparison of the mean lengths of normal $(\mathrm{N})$ and reversed $(\mathrm{R})$ polarity intervals and the values of the gamma index ( $k$ ) for several magnetic polarity time scales. The comment in the $(*)$ column indicates whether the time series is stationary

\begin{tabular}{|c|c|c|c|c|c|c|c|c|c|c|c|}
\hline \multirow[t]{2}{*}{ Time scale } & \multirow{2}{*}{$\begin{array}{l}\text { Age range } \\
\text { (Myr) }\end{array}$} & \multicolumn{3}{|c|}{ Number of intervals } & \multicolumn{3}{|c|}{ Mean length } & \multicolumn{3}{|c|}{ Gamma index, $k$} & \multirow[t]{2}{*}{$(*)$} \\
\hline & & all & $\mathbf{N}$ & $\mathbf{R}$ & all & $\mathbf{N}$ & $\mathbf{R}$ & all & $N$ & $\mathbf{R}$ & \\
\hline Heirtzler et al. [1] & $0-48$ & 142 & 71 & 71 & 0.338 & 0.332 & 0.343 & 1.57 & 2.40 & 1.18 & yes \\
\hline Lowrie and Alvarez [4] & $0-40$ & 124 & 62 & 62 & 0.322 & 0.292 & 0.351 & 1.50 & 1.82 & 1.32 & no \\
\hline LaBrecque et al. [2] & $0-40$ & 126 & 63 & 63 & 0.314 & 0.285 & 0.344 & 1.52 & 1.90 & 1.28 & no \\
\hline \multicolumn{12}{|l|}{ LaBrecque et al. [2] with } \\
\hline 30-kyr events & $0-40$ & 226 & 113 & 113 & 0.175 & 0.166 & 0.184 & 1.42 & 1.14 & 2.01 & yes \\
\hline
\end{tabular}

\section{Short duration polarity intervals}

It appears that, except for the last $10 \mathrm{Myr}$ or so, the lengths of magnetic polarity intervals fit a gamma distribution better than a Poissonian distribution. The gamma model implies that short polarity intervals never occurred.

An alternative explanation is that the short polarity intervals did indeed occur but that they can not readily be resolved in ocean-surface magnetometer surveys. Continental magnetostratigraphic investigations of the oceanic reversal sequence do not clarify this point, because the resolution of such studies $(30-100 \mathrm{kyr})$ is not better than that of an oceanic magnetic survey.

Blakely and Cox [14] enhanced the coherency of six marine magnetic profiles from the North Pacific ocean by using a stacking procedure, and detected six new short-wavelength anomalies between the Early Tertiary anomalies 21 and 29. Blakely [15] applied the same technique to fourteen marine magnetic profiles from the northeast Pacific and identified eight new short-wavelength anomalies. These small coherent anomalies have been interpreted as related to hitherto undetected, short polarity chrons.

An alternative interpretation of the coherent, short wavelength anomalies is that they represent geomagnetic intensity fluctuations [16]. The ambiguous nature of very short wavelength magnetic anomalies prompted LaBrecque et al. [2] to omit most of them from their tabulated list of polarity interval ages. However, the times of occurrence of the possible short events were indicated in a chart of the polarity time scale [2, fig. 1]. The mid-point ages of the events can be estimated between polarity chron boundaries, assuming linearity of spreading between these limits. We have investigated the consequences of including them as short polarity chrons in the LaBrecque et al. [2] polarity time scale.

\section{The effect of adding short events to the mag- netic polarity time scale}

An accurate estimate of the mid-point ages is important because each new event subdivides the polarity interval in which it occurs, which has an important effect on the histogram of polarity interval lengths [5]. The exact duration of each event can not be determined as it is too close to the limit of resolution of the oceanic record. Most likely they are not longer than about $40 \mathrm{kyr}$ and so we have investigated the effects of assigning the events equal durations of 20,30 or $40 \mathrm{kyr}$.

Regardless of which of these durations is chosen, the short events have a dramatic effect on the polarity interval distribution for the $0-40 \mathrm{Myr}$ age interval. The number of polarity intervals increases from 126 to 226 and the mean length of a polarity interval is reduced from 0.314 to 0.175 Myr. The slope of the linear regression of polarity interval length against age no longer differs significantly from zero at the $95 \%$ confidence level (Fig. 4); the polarity interval distribution is now stationary and can be analysed further statistically. 


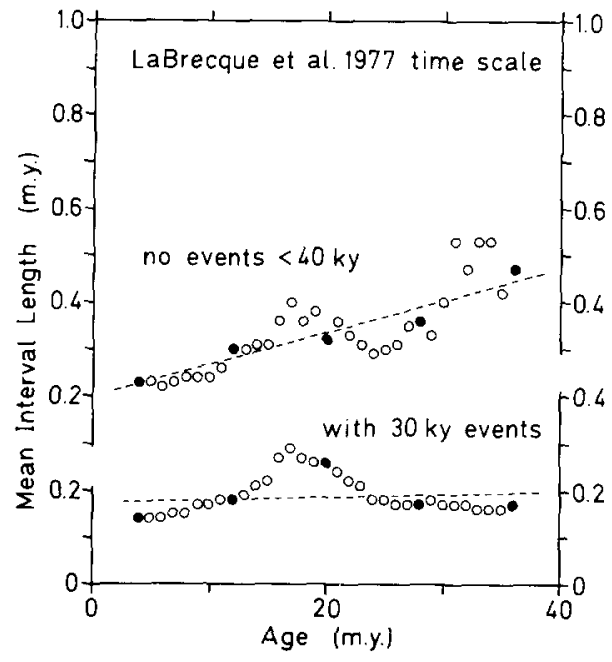

Fig. 4. The average duration of a polarity chron in the interval $0-40 \mathrm{Myr}$ of the LaBrecque et al. [2] time scale shows a linear trend with age. The slope of the linear regression of every eighth point with age is significant and the time series is not stationary. After adding fifty $30-\mathrm{kyr}$ polarity chrons the slope is no Jonger significant and the time series is now stationary.

The choice of duration for the short chrons does, however, influence the ability of a Poissonian distribution to fit the polarity interval length distribution (Fig. 5). When the short chrons are allotted equal lengths of $20 \mathrm{kyr}$, the observed distribution is found by a chi-square test to differ significantly from the Poissonian distribution. However, if the short chrons are given equal lengths of $30 \mathrm{kyr}$, the discrepancy is no longer significant at the $95 \%$ confidence level.

The effect of additional short chrons is seen dramatically in the distributions of normal and reversed polarity interval lengths. The mean durations are reduced to $0.166 \mathrm{Myr}$ and $0.184 \mathrm{Myr}$, respectively. The gamma index, $k$, is now visibly different for the distributions of normal and reversed intervals (Fig. 6).

The choice of length for the short events also affects the values of $k$ obtained for the normal and reversed distributions, although in each case $k$ for normal polarities is smaller than $k$ for negative polarities. If the events are assigned the very short duration of $20 \mathrm{kyr}$, the value of $k$ for normal polarity intervals is less than 1 . This case must be excluded as it would predict an infinite population. By implication the interpretion of all shortwavelength magnetic anomalies as polarity chrons is not justified, or the duration assigned to them is incorrect. When the short chrons are assigned equal durations of $30 \mathrm{kyr}$, the values obtained for the gamma indices are 1,14 and 2.01 for the normal and reversed distributions, respectively. With equal durations of $40 \mathrm{kyr}$ gamma indices of 1.32 and 2.12 are obtained.

We note that adding the maximum possible

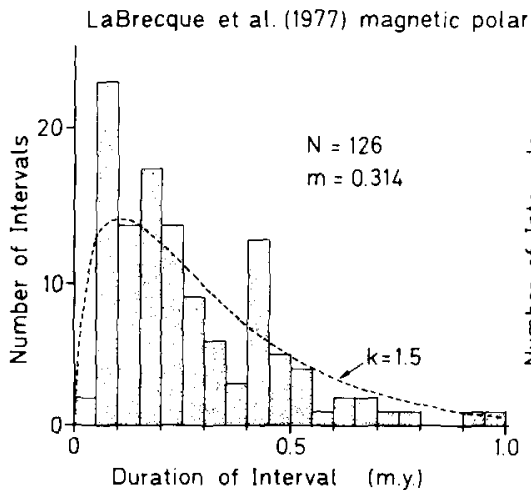

(a)

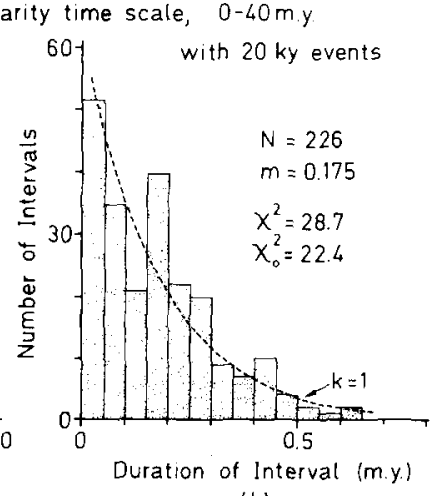

(b)

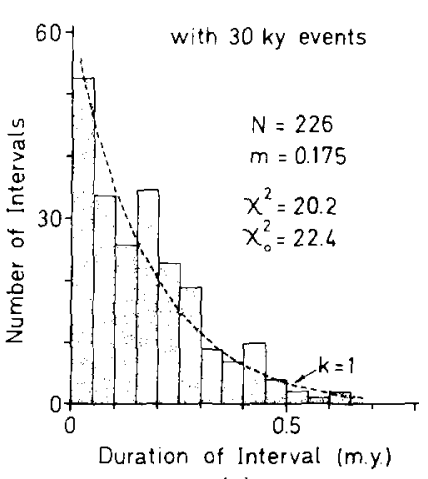

(c)

Fig. 5. (a) The lengths of polarity intervals in the 0-40 Myr part of the LaBrecque et al. [2] magnetic time scale resemble a gamma distribution with index 1.5 (strictly speaking, the series is not stationary). (b) When 20-kyr short polarity chrons are added, the chi-square test shows that the distribution approximates to a Poisson distribution but differs significantly from it. (c) When the added short polarity chrons are given duration $30 \mathrm{kyr}$, the distribution of polarity interval lengths does not differ significantly from a Poisson distribution. 
LaBrecque et al. 1977 time scale

$0-40$ my. with 30 ky events
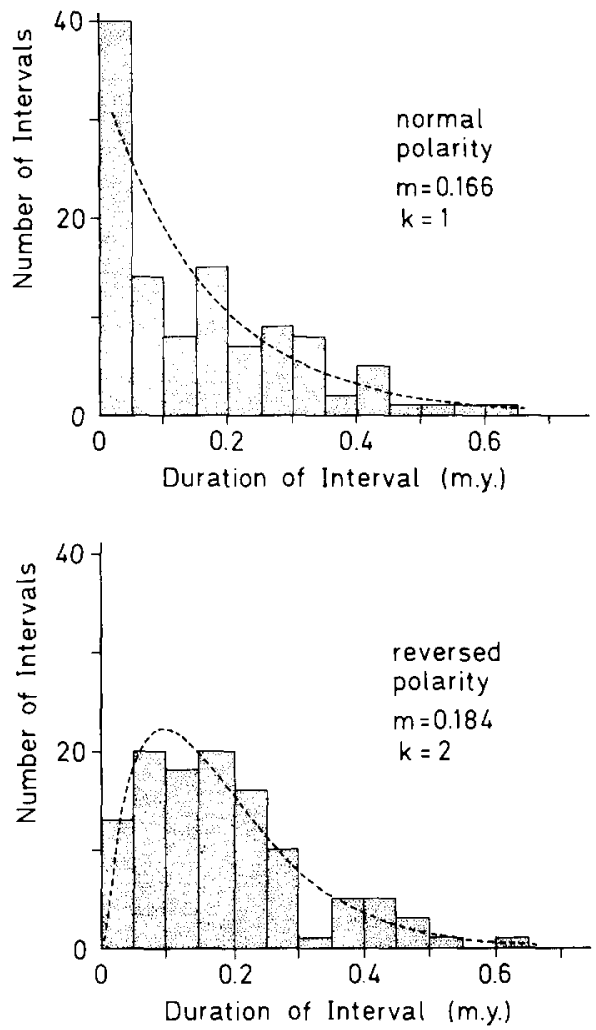

Fig. 6. The 0-40 Myr part of the LaBrecque et al. [2] time scale to which 50 short polarity chrons of equal duration $30 \mathrm{kyr}$ have been added shows asymmetry between the distribution of normal polarity chrons (approximately Poissonian, $k=1$ ) and reversed polarity chrons (approximately gamma distributed, $k=$ 2).

number of short events to the LaBrecque et al. [2] polarity time scale gives a mean duration for normal polarity chrons less than that for negative polarity chrons, as was also observed in analyses of the superseded Heirtzler et al. [1] time scale $[6,7]$. However, the gamma index for normal polarity chrons is now smaller than that for negative polarity chrons. The added short events have altered the sense of the asymmetry between the normal and reversed states.

\section{Discussion}

A question of major importance for statistically modelling magnetic field polarity reversals is the origin of the short-wavelength magnetic anomalies that have been observed as "tiny wiggles" on high-resolution marine magnetic profiles. Comparatively few of them have been investigated thoroughly, thus it is not known how many of the 57 possible small events indicated in the LaBrecque et al. [2] time scale are coherent. There is ambiguity as to the interpretation of coherent short-wavelength anomalies as polarity chrons $[14,15]$ or as magnetic field intensity fluctuations [16].

If all postulated short polarity chrons are real, they have a strong influence on the statistical properties of the magnetic polarity time scale. They result in stationarity in the age range $0-40$ Myr of the LaBrecque et al. [2] magnetic polarity time scale, where this was previously absent. The distribution of all polarity intervals (Fig. 5) in this age range becomes Poissonian and the normal and reversed polarity interval distributions acquire a strong asymmetry in the opposite sense to the distributions without short polarity chrons. This is due to the unequal distribution of the 57 short polarity chrons over the Cenozoic part of the LaBrecque et al. [2] time scale. They are overwhelmingly of positive polarity and are most abundant after the end of the Eacene, 50 of them being younger than $40 \mathrm{Myr}$.

This unequal distribution also requires explanation. Possibly the predominance of young events arises from a bias of marine magnetic data to regions on or near spreading ridge systems. Shortwavelength anomalies also may be more difficult to resolve over older ocean floor, due for example to the greater anomaly source depth.

The interpretation of coherent short-wavelength anomalies as magnetic field phenomena has also been questioned. Schouten and Denham [17] developed a sophisticated model for generation of new oceanic crust at a spreading center which allows representation of high spatial complexity of the magnetized layer yet reproduces the observed magnetic reversal sequence. They draw attention to the possibility that short-wavelength magnetic anomalies may result from variability in the magnetized layer in high-spreading-rate ridge systems. The prevalence of short events within negative polarity chrons may have a rock magnetic origin, such as induced or viscous magnetization. 
It is evident that the origin of these short features is questionable on several grounds. They may represent polarity chrons, intensity fluctuations or source-layer variations. Until the ambiguity is resolved their interpretation as part of the geomagnetic polarity record must be treated with caution and reserve.

The reversal records which we accept as best representative of geomagnetic field behavior are the LaBrecque et al. [2] and Lowrie and Alvarez [4] time scales. The second is tied in better than the first to the biostatigraphic record. The statistical properties of these two polarity time scales are very similar but differ substantially from the Heirtzler et al. [1] time scale. A major difference is the lack of stationarity in the newer time scales in the interval since anomaly 18 (which was dated as about $48 \mathrm{Myr}$ in Heirtzler et al. [1] and closer to 40 Myr in the newer time scales). Although strictly the statistical analysis is only of qualitative value, the 0-40 Myr polarity intervals conform closely to a gamma distribution with index close to 2 . This would imply, if true, that the probability of a reversal per unit time is not constant as assumed in the stochastic model of Cox [5,13].

Another difference is that the discontinuity separating two stationary domains in the Heirtzler et al. [1] time scale is much less apparent in later time scales; it arises primarily from inclusion of the non-existent anomaly 14 . The change in reversal frequency with time (Fig. 1) is best represented as a linear trend on which is superposed a rhythmic fluctuation with an apparent periodicity of around $20 \mathrm{Myr}$. These features may represent secular changes in core conditions during the past $80 \mathrm{Myr}$ and indicate that core processes may have a longterm memory for polarity reversals.

\section{Conclusions}

As earlier investigators have concluded, the statistical properties of geomagnetic polarity reversals are strongly influenced by the existence of short events. The source of short-wavelength marine magnetic anomalies is controversial; they could represent geomagnetic intensity changes, short polarity events or source-layer inhomogene- ity. The dilemma can only be solved by detailed marine magnetic profiling with deep-tow techniques.

The distribution of "tiny wiggles" which can be interpreted as short events is very uneven, and most of them are of positive polarity. If the postulated events are real, the asymmetry implies that the geomagnetic field is more stable in one polarity state than the other. This may reflect different mechanisms for the normal-reversed and reversed-normal transitions.

If the "tiny wiggles" do not represent short polarity events, the distribution of polarity intervals is not Poissonian. This implies that the probability of a reversal per time is not constant, but that core processes have a long-term memory for polarity reversals. The geomagnetic field is then not characterized by lengthy periods of stationary behavior. Instead, the average reversal frequency shows a rhythmic fluctuation superposed on a linear trend. These indicate secular changes in the core conditions governing reversal processes.

\section{Acknowledgements}

We thank S. Cande, F. Heller and J. LaBrecque for their criticisms of the manuscript. Financial support from N.S.F. grant OCE81-19695 and the E.T.H.-Zürich is gratefully acknowledged.

\section{References}

1 J.R. Heirtzler, G.O. Dickson, E.M. Herron, W.C. Pitman III and X. Le Pichon, Marine magnetic anomalies, geomagnetic field reversals and motion of the ocean floor and continents, J. Geophys. Res. 73 (1968) 2119-2136.

2 J.L. LaBrecque, D.V. Kent and S.C. Cande, Revised magnetic polarity time scale for Late Cretaceous and Cenozoic time, Geology 5 (1977) 330-335.

3 G. Ness, S. Levi and R. Couch, Marine magnetic anomaly timescales for the Cenozoic and Late Cretaceous: a precis, critique and synthesis, Rev. Geophys. Space Phys. 18 (1980) 753-770.

$4 \mathrm{~W}$. Lowrie and W. Alvarez, 100 million years of geomagnetic polarity history, Geology 9 (1981) 392-397.

5 A. Cox, Geomagnetic reversals, Science 163 (1969) 237-245.

6 P.S. Naidu, Statistical structure of geomagnetic field reversals, J. Geophys. Res. 76 (1971) 2649-2662. 
7 J.D. Phillips, Time variation and asymmetry in the statistics of geomagnetic reversal sequences, J, Geophys. Res. 82 (1977) 835-843.

8 A. Cox, The frequency of geomagnetic reversals and the symmetry of the nondipole field, Rev. Geophys. Space Phys. 13 (1975) 35-51.

9 W. Lowrie and W. Alvarez, Upper Cretaceous-Paleocene magnetic stratigraphy at Gubbio, Italy, III. Upper Cretaceous magnetic stratigraphy, Geol. Soc. Am. Bull. 88 (1977) 374-377.

$10 \mathrm{~W}$. Lowrie and W. Alvarez, Late Cretaceous geomagnetic polarity sequence: detailed rock and paleomagnetic studies of the Scaglia Rossa limestone at Gubbio, Italy, Geophys. J. R. Astron. Soc. 51 (1977) 561-582.

11 S.C. Cande and Y. Kristoffersen, Late Cretaceous magnetic anomalies in the North Atlantic, Earth Planet. Sci. Lett. 35 (1977) 215-224.

12 W. Lowrie, W. Alvarez, G. Napoleone, K. Perch-Nielsen, I. Premoli Silva and M. Toumarkine, Paleogene magnetic stratigraphy in Umbrian pelagic carbonate rocks: the Contessa sections, Gubbio, Geol. Soc. Am. Bull. 93 (1982) 414-432.

13 A. Cox, Lengths of geomagnetic polarity intervals, J. Geophys. Res. 73 (1968) 3247-3260.

14 R. Blakely and A. Cox, Evidence for short geomagnetic polarity intervals in the Early Cenozoic, J. Geophys. Res. 77 (1972) 7065-7072.

15 R.J. Blakely, Geomagnetic reversals and crustal spreading rates during the Miocene, J. Geophys. Res. 79 (1974) 2979-2985.

16 S.C. Cande and J.L. LaBrecque, Behaviour of the earth's palaeomagnetic field from small scale marine magnetic anomalies, Nature 247 (1974) 26-28.

17 H. Schouten and C.R. Denham, Modelling the oceanic magnetic source layer, in: Deep Drilling in the Atlantic Ocean: Ocean Crust, M. Talwani, C.G. Harrison and D.E. Hayes eds., Am. Geophys. Union, Maurice Ewing Ser. 2 (1979) 151-159. 